\title{
Salud y crisis
} de equidad) de las crisis económicas sobre la salud general.

La crisis económica y financiera llega a las finanzas públicas de forma abrupta en 2008: en 2007 hubo un superávit del 1,9\% del PIB, pero en 2008 cae un 6,4\%, y en 2009 otro 6,7\% (con la cifra récord de déficit del-11,2\%). No obstante, el sistema de financiación autonómica contaba con un sistema de "pagos a cuenta" que retrasó la reducción de ingresos autonómicos y amortiguó la llegada de la crisis.

En 2010 la situación se expresa con toda la crudeza, y es entonces cuando se activan tardíamente las respuestas de ajuste. Entre estas respuestas caben destacar: cambios en el modelo sanitario (aseguramiento RD 16/2012); revisión de la cartera de servicios; nuevos copagos; exclusión de la financiación de medicamentos; cambios en el modelo de gestión y recortes presupuestarios (cierres de camas hospitalarias, contratos parciales de profesionales sanitarios, incrementos de las listas de espera quirúrgicas y en especialidades, demoras en AP y pruebas diagnósticas, saturaciones en servicios de urgencias, cierres de PAC y de horarios de tarde en centros de salud...).

$\mathrm{Si}$ a todos estos recortes o "ajustes" le sumamos las otras consecuencias de la crisis económica (alta tasa de desempleo, precariedad en algunos puestos de trabajo -también entre los profesionales sanitarios-, disminución salarial -relativa y absoluta-, alta cuota de jóvenes desempleados y claramente preparados, etc.) pretender que todo ello sumado y aderezado por nuestros políticos no afecte a la salud de la población no deja de ser falaz e irrisorio.

Como "muestra: 2 botones", en el magnífico artículo que salió publicado en el número anterior de la revista (Rev Soc Esp Dolor 2015;22(2):51-8) se presentó “Crisis económica española y salud. Impacto clínico en el dolor crónico" del que más adelante expresaré mi opinión y las referencias y evidencias en cuanto a que la salud mental se ve negativamente afectada en los periodos de recesión económica (1).

Las crisis económicas se sabe bien que ejercen un impacto negativo sobre la salud mental por diversos factores interrelacionados, entre los que el desempleo constituye el principal factor de riesgo. Numerosos estudios han utilizado esta variable para investigar la relación entre salud mental y crisis económica (2-6). Perder el trabajo y pasar a la situación de paro tiene consecuencias sobre la salud de las personas, sobre todo en la esfera psicosocial (7-9). Las diferentes publicaciones que analizan el bienestar afectivo de empleados y desempleados muestran una mayor presencia de sintomatología ansiosa y depresiva entre las personas sin empleo. También es importante recordar que hay estudios que ponen de manifiesto que las prestaciones económicas de desempleo evitan o reducen este efecto y que las consecuencias negativas sobre la salud se centran en las personas sin prestaciones (10-16).

Una de las esferas menos analizadas hasta ahora ha sido la relación entre crisis económica (recortes) y dolor crónico, en el excelente artículo presentado en el número anterior de la revista (Rev Soc Esp Dolor 2015;22(2):51-8) no sólo se pone de manifiesto la relación o asociación entre 
dolor y crisis, sino que se da un paso más en lo referente a un análisis en la equidad (más bien inequidad) producida por las reformas, y de cómo estas (como casi siempre suele ocurrir) afectan a los más débiles y/o desprotegidos. También se analiza y discute sobre los determinantes sociales de la salud, en concreto educación y asistencia sanitaria.

No es aceptable que en nuestro medio, para evitar la adopción de decisiones de priorización explícita y responsable, se establezcan recortes uniformes y lineales, es decir a todos por igual, ocurriendo que quien más lo sufre sea quien mejor lo hace.

Los autores del trabajo además concluyen con la parte positivista de toda crisis, oportunidad de cambio, como debería ser, a las que se podrían añadir otras (17): desinvertir en lo que no añade salud, prioridad clínica y transparencia en la gestión de las listas de espera, reformar el inequitativo sistema de copagos, retribución según resultados y esfuerzos, más y mejor salud pública, cambio de foco hacia pacientes crónicos, profesionalizar la gestión (que desaparezcan los puestos gerenciales como botín electoral), mejorar la información para comparar, etc.

Mis más sincera enhorabuena a los autores de este buen trabajo.

A.J. García Ruiz

Cátedra de Economía de la Salud. Departamento de Farmacología. Facultad de Medicina. Universidad de Málaga. Málaga

\section{BIBLIOGRAFÍA}

1. Gili M, García Campayo J, Roca M. Crisis económica y salud mental. Informe SESPAS 2014. Gac Sanit 2014;28(S1):104-8.

2. Chang S, Gunnell D, Sterne J, et al. Was the economic crisis 1997-1998 responsible for rising suicide rates in East/Southest Asia? A time-trend analysis for Japan, Hong Kong, South Korea, Taiwan, Singapore and Thailand. Soc Sci Med 2009;68:1322-31.

3. Lee S, Guo WJ, Tsang A, et al. Evidence for the 2008 economic crisis exacerbating depression in Hong Kong. J Affect Disord 2010;126:125-33.

4. Chen L, Li W, He J, et al. Mental health, duration of unemployment, and coping strategy: a crosssectional study of unemployed migrant workers in eastern China during the economic crisis. BMC Public Health 2012. Disponible en: http://www.ncbi.nlm.nih.gov/pmc/articles/PMC3490784/

5. Hong J, Knapp M, McGuire A. Income-related inequalities in the prevalence of depression and suicidal behaviour: A 10-year trend following economic crisis. World Psychiatry 2011;10:40-4.

6. Stuckler D, Basu S, Suhrcke M. The public health effect of economic crises and alternative policy responses in Europe: an empirical analysis. Lancet 2009;374:315-23.

7. Bartley M. Unemployment and ill health: understanding the relationship. J Epidemiol Comm Health 1994;48:333-7.

8. Janlert U. Unemployment as a disease and diseases of the unemployed. Scand J Work Environ Health 1997;23(Supl. 3):79-83.

9. Dooley D, Fielding J, Levi L. Health and unemployment. Annu Rev Public Health 1996;17:449-65.

10. Leeflang RLI, Klein-Hesselink J, Spruit P. Health effects of unemployment-II. Men and women. Soc Sci Med 1992;34:351-63.

11. Kessler RC, Turner JB, House JS. Effects of unemployment on health in a community survey: Main, modifying and mediating effects. J Soc Issues 1988;34:341-50.

12. Rodgers B. Socio-economic status, employment and neurosis. Soc Psychiatry Epidemiol 1991;26:104-14.

13. Rodríguez E, Lasch K, Mead JP. The potential role of unemployment benefits in shaping the mental health impact of unemployment. Int J Health Serv 1997;27:601-23.

14. Rodríguez E, Frongillo EA, Chandra P. Do social programmes contribute to mental well-being? The long- term impact of unemployment on depression in the United States. Int J Epidemiol 2001;30:6370.

15. Rodríguez E. Keeping the unemployed healthy: The effect of means tested and entitlement benefits in Britain, Germany, and the United States. Am J Public Health 2001;91:1403-11.

16. Artazcoz L, Benach J, Borrell C, Cortès I. Unemployment and mental health: Understanding the interactions among gender, family roles, and social class. Am J Public Health 2004;94:82-8.

17. Documento de debate. Asociación de Economía de la Salud. La Sanidad Pública ante la crisis. Recomendaciones para una actuación pública sensata y responsable. Disponible en: http://www.aes.es/ Publicaciones/DOCUMENTO_DEBATE_SNS_AES.pdf. Accedido el 16 de marzo de 2015. 